\title{
Roadmap to high-energy square pulses in anomalous dispersion fiber lasers
}

\author{
G. Semaan, ${ }^{* 1}$ F. Ben Braham, ${ }^{1,2}$ M. Salhi ${ }^{1}$ and F. Sanchez ${ }^{1}$ \\ ${ }^{1}$ Laboratoire de Photonique d'Angers E.A 4464, Université d'Angers, 2 Bd Lavoisier49045, Angers, France, \\ ${ }^{2}$ Laboratoire Systèmes Electroniques et Réseaux de Communications (SERCOM), Ecole polytechnique de Tunisie, \\ EPT B.P 743, 2078, Université de Carthage, Tunisia
}

Received October 30, 2016; accepted November 23, 2016; published December 31, 2016

\begin{abstract}
Dissipative soliton resonance (DSR) is a soliton formation where the energy in a dissipative system becomes infinite. In the anomalous dispersion regime, this energy is not limited by the soliton area theorem. Since this phenomenon is wave breaking free, it can be useful in designing fiber lasers generating pulses with relatively high energies. Based on this principle, we have demonstrated the emission of high energetic square pulses from Er: $\mathrm{Yb}$ double-clad passively modelocked fiber lasers using different mode-locking mechanisms. We first show the evolution of pulse width, energy and peak power by varying the pumping power of the amplifier, then we control separately the characteristic of the output square pulse by assigning each one to an amplifier. Experimental results exhibit record energies in fiber lasers up to $10 \mu \mathrm{J}$.
\end{abstract}

The development of fiber laser technology and their multiple applications has stimulated a great interest in investigating novel operational modes such as highenergy and wide width pulses. Since high energy square pulses have applications in different fields such as optical sensors, square-wave clocks and biomedical progress, they have been widely explored. However, it has been a difficult task to achieve high pulse energies because of different limiting factors such as multi-pulsing instabilities that occur on high pumping power. Indeed, in the anomalous dispersion regime the pulse energy is limited by the soliton area theorem while in a normal dispersion regime it is the nonlinear losses combined with the finite gain bandwidth which limit the energy of the pulses. The limitation manifests itself by the emission of additional pulses when the pumping power increases. To overcome these problems, different strategies have been developed like beam shaping. Different kinds of a pulse shape, such as a parabolic pulse, and a square pulse have been generated to satisfy different demands. Achieving high energy square pulses can be realized by exploiting the dissipative soliton resonance (DSR) theory [1], where the energy is not limited by the soliton area theorem and it increases linearly, which allows the pulse width to widen linearly.

The first theoretical study of the DSR phenomenon was based on the complex cubic-quintic Ginzburg-Landau equation [1]. It predicts that the soliton energy increases

${ }^{*}$ E-mail: georges.semaan@univ-angers.fr indefinitely with specific parameters while keeping the peak power stable at a certain level. The normal dispersion regime favors larger pulse energy compared to the anomalous dispersion regime [2]. An interesting property is that the energy of the square pulse increases proportionally with the pumping power which allows the pulse duration to widen linearly with the pump power. The square pulses were experimentally observed in setups consisting of the nonlinear polarization evolution mechanism in normal [3] and anomalous dispersion regime [4]. The generation of the DSR square pulse has also been demonstrated in figure-of-eight fiber lasers using optical circulators [5] and dual pumping [6]. It has been shown experimentally that high non-linearity plays an important role in the widening pulse [7]. Recently, dissipative soliton square pulses were demonstrated in a novel figure-9 fiber laser configuration with a pulse duration recorded around $455 \mathrm{~ns}$ and an output energy per pulse about $2.3 \mu \mathrm{J}[8]$.

In this letter, we present, in different configurations, an experimental study on achieving up to $10 \mu \mathrm{J}$ pulse energy $[4,9]$ for a square pulse operating in a dissipative soliton resonance region, with tunable width in a wide range.

Since long fibre cavities were experimentally used in order to favour the generation of high energy square pulses under the DSR regime [10], we have built long cavities operating in an anomalous dispersion regime relaying on nonlinear polarization evolution and figureof-eight mode-locking mechanisms. The first demonstration consists in a simple all fibre ring cavity passively mode-locked through nonlinear polarization evolution. The total cavity length is about $536 \mathrm{~m}$ with a total dispersion of $\beta_{2}^{\text {Total }} \times L=-11.872 \mathrm{ps}^{2}$. The $2.681 \mu \mathrm{s}$ round-trip time of the cavity corresponds to a free spectral range of $373 \mathrm{kHz}$. Two polarization controllers are used to control nonlinear losses. A polarization-insensitive isolator (PI-ISO) is employed to force unidirectional operation of the cavity and a 10/90 output coupler is used to extract $10 \%$ of the power from the cavity. The operating $10 \mathrm{~W}$ amplifier is a double-clad V-groove Er:Yb 10W fiber amplifier manufactured by Keopsys and has a security requirement for protecting the 
internal circuitry which requires an external optical threshold input signal to start. This signal is delivered by a homemade continuous wave tunable fibre laser and is directly injected in the cavity through a 3-dB fibre coupler. The external laser is switched off once the $10-\mathrm{W}$ amplifier starts operating. The external laser is switched off once the $10 \mathrm{~W}$ starts operating.

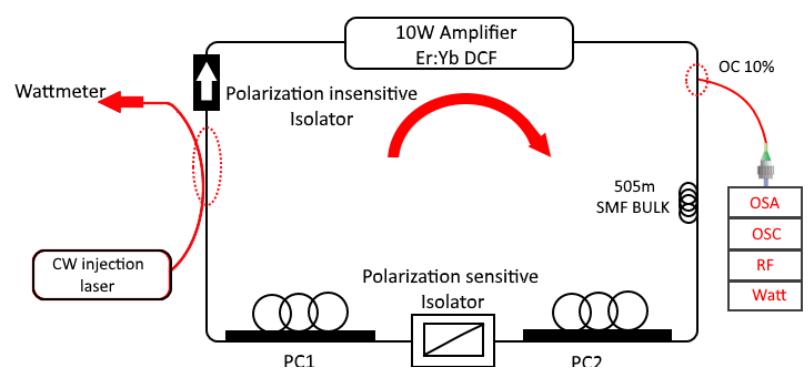

Fig. 1. Experimental setup of the ring cavity.

In both configurations presented in this letter, the output intensity is measured using a high-speed photodetector (TIA-1200), and visualized with a fast oscilloscope (Tektronix TDS 6124C, $12 \mathrm{GHz}, 40 \mathrm{GSa}$ ). Also, the output power is measured using a high-power wattmeter detector (Coherent Molectron PM500AD). The spectral properties are analysed with an optical spectrum analyser (Anritsu MS 9710C).

The DSR regime is obtained by carefully adjusting the polarization controllers. Figure 2 exhibits the evolution of pulses as a function of the pumping power. While the pump power is increased, the amplitude of the square pulses seems to remain nearly constant and the pulse duration increases without any wave breaking in contrast to the standard soliton regime.

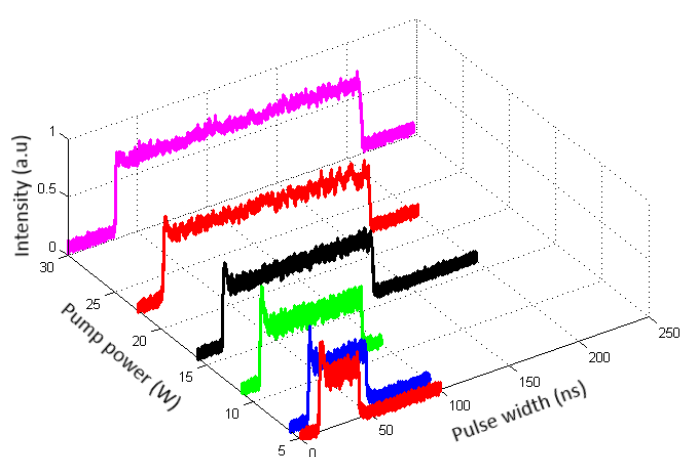

Fig. 2. Evolution of the generated DSR square-wave pulse versus the pumping power.

To confirm the DSR regime, we have checked that the evolution of both the pulse duration and the pulse energy are linearly dependent on the pumping power and that the peak power remains constant. The experimental results

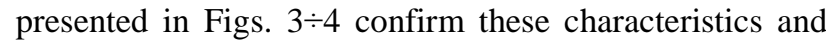
prove that the laser operates in the DSR regime. We have noticed that the pulse energy and duration are not continuously linear over the complete pumping power range but in fact are linear in certain ranges. This is due to the fact that the $10 \mathrm{~W}$ amplifier used in this experiment has 2 distinct operating regimes: the low power regime requires a minimum input of $17 \mathrm{dBm}\left(P_{\text {pump }} \leq 7 \mathrm{~W}\right)$ and the high-power regime for which the gain of the amplifier must be below $13 \mathrm{~dB}\left(7 \leq P_{\text {pump }} \leq 26 \mathrm{~W}\right)$.

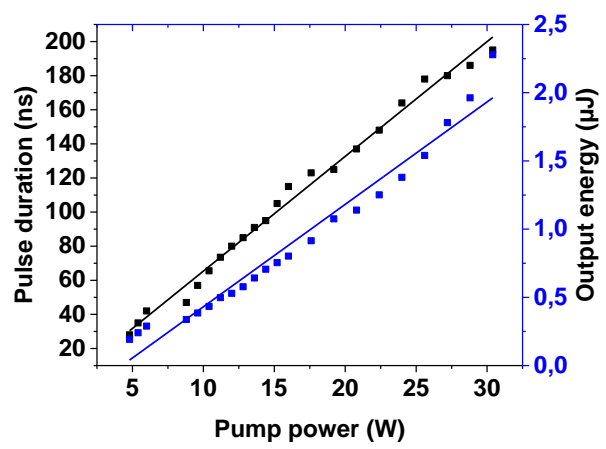

Fig. 3. Evolution of pulse duration and energy vs pump power.

For a pump power over $26 \mathrm{~W}$, multi-pulsing instability emerges, which requires adjustment of the polarization controllers to remove it, and thus alteration of the output pulse characteristics.

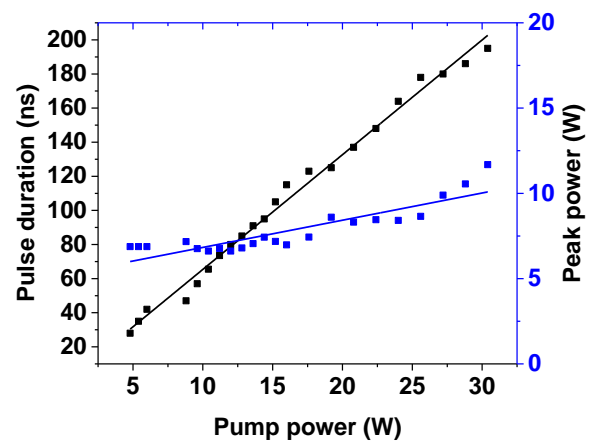

Fig. 4. Evolution of pulse duration and peak power vs pump power.

The highest achieved pulse energy is about $2.3 \mu \mathrm{J}$ and is connected with the output coupling coefficient used for the extraction of the optical power from the cavity. As shown in Fig. 2, the achieved generated square-wave pulses are tuned in a range from 28ns to 190ns. It is worth mentioning that during the tuning in our experiment there was no pulse breaking, nor pulse bunching or higher harmonic operation till we passed the $26 \mathrm{~W}$ threshold of the pump power.

In the second experimental setup shown in Fig. 5, we built a cavity based on an all-fiber F8L that consists in a main unidirectional ring cavity (UR) connected to a nonlinear amplifying loop mirror NALM by a fused fibre coupler. 


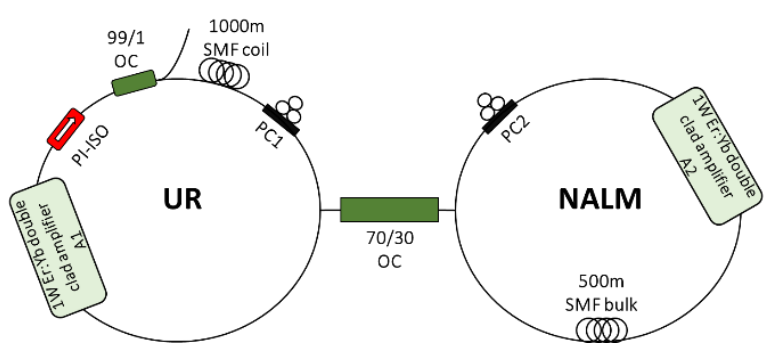

Fig. 5. Experimental setup of the figure-of-eight cavity.

In the main cavity, we use a $30 \mathrm{dBm}$ C-band double-clad co-doped fiber amplifier (A1) with a pump power of $3 \mathrm{~W}$ at $980 \mathrm{~nm}$. The polarization insensitive isolator (PI-ISO) is inserted between the amplifier and the output coupler to block the counter-clockwise wave. A $1 \mathrm{~km}$ SMF coil is inserted after the amplifier to increase the nonlinearities and the round-trip time of the cavity. In the NALM, we have another $30 \mathrm{dBm} \mathrm{C}$-band double-clad co-doped fiber amplifier (A2) with a pump power of $5 \mathrm{~W}$ at $980 \mathrm{~nm}$. A $500 \mathrm{~m}$ SMF coil is inserted after the amplifier to increase the asymmetric nonlinear phase shift between the clockwise and counter-clockwise propagating light in the NALM. Two polarization controllers are used to adjust the polarization states. The total cavity length is about $1536 \mathrm{~m}$; the net cavity dispersion is about $-33 \mathrm{ps}^{2}$ and the round-trip time is $7.65 \mu \mathrm{s}$ corresponding to a free spectral range of $133 \mathrm{kHz}$. A $99 / 1$ fiber output coupler is used to extract $99 \%$ of the power in the cavity.

The DSR regime is obtained by carefully adjusting the paddles of the polarization controllers while pumping A1 with $500 \mathrm{~mW}$ and $\mathrm{A} 2$ with $700 \mathrm{~mW}$. At a maximum pumping power in $\mathrm{A} 1$ of $3 \mathrm{~W}$ and $\mathrm{A} 2$ of $1.7 \mathrm{~W}$ we obtain a stable single square pulse in the cavity with an output power of $1.33 \mathrm{~W}$ for a roundtrip of $7.65 \mu \mathrm{s}$ leading us to a $10 \mu \mathrm{J}$ energy per pulse. The experimental results in Fig. 6 and Fig.7 show the evolution and dependency of pulse characteristics as a function of pumping power.

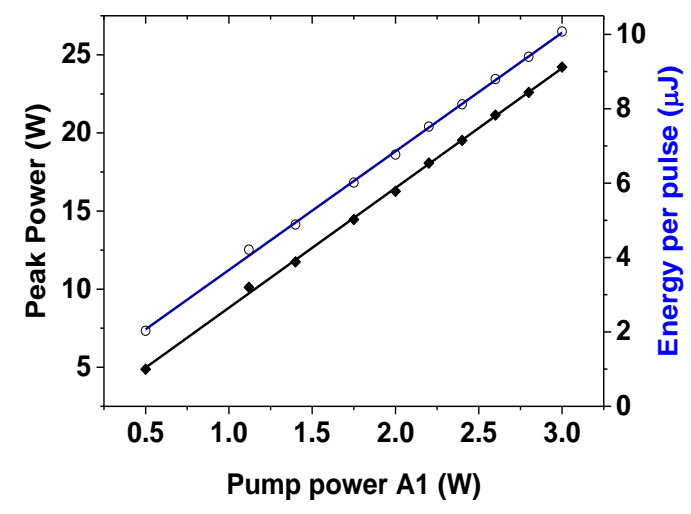

Fig. 6. Experimental setup of the figure-of-eight cavity.

By fixing A2 and while the pump power of A1 is increased, the amplitude and output energy increase linearly while the pulse width remains fairly constant at 416ns. In contrast to the results in [6], we now set the pumping power of $\mathrm{A} 1$ at a non-zero value and we tune the pumping power of A2. The square pulses width increases linearly as predicted for the DSR pulses but since the peak power is decreasing while the width is increasing, this results in a slow increase of the output energy. Thus, for the set of parameters established in this experiment, the operation of both amplifiers is not fully independent but can be if A1 is completely turned off.

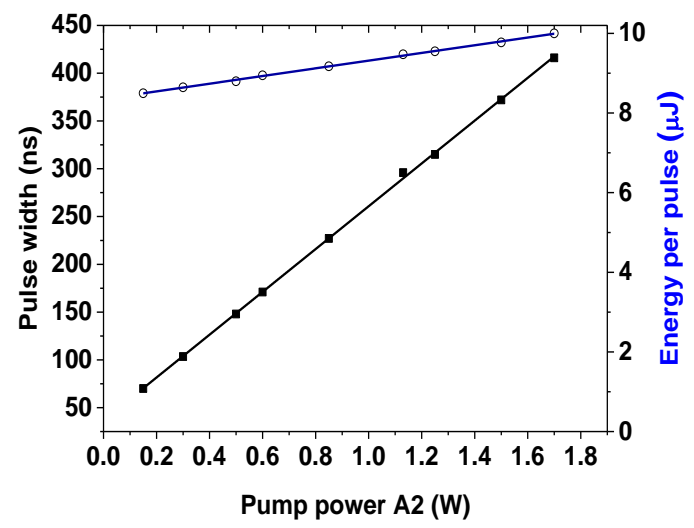

Fig. 7. Experimental setup of the figure-of-eight cavity.

In conclusion, in this letter we have presented the generation of high energy square pulses operating in the DSR regime using a different mode-locking mechanism. The pulse width and energy are linear with the pump power while maintaining a constant peak power. We have also shown that the pulse characteristics can be controlled in a figure-of-eight laser cavity by using two amplifiers. The achieved output energies shown in this letter present a $3 \%$ slope efficiency under nonlinear polarization evolution due to the existence of a $10 \%$ output coupler and a $29 \%$ slope efficiency in a double amplifier configuration.

\section{References}

[1] W. Chang, A. Ankiewicz, J.M. Soto-Crespo, N. Akhmediev, Phys. Rev. A 78, 023830 (2008).

[2] P. Grelu, W. Chang, A. Ankiewicz, J.M. Soto-Crespo, N. Akhmediev, J. Opt. Soc. Am. B 27, 2336 (2010).

[3] X. Wu, D.Y. Tang, H. Zhang, L.M. Zhao, Opt. Expr. 17, 5580 (2009).

[4] G. Semaan, F. Ben Braham, M. Salhi, Y. Meng, F. Bahloul, F. Sanchez, Opt. Expr. 24, 8399 (2016).

[5] K. Krzempek, Opt. Expr. 23, 30651 (2015).

[6] L. Mei et al., Opt. Lett. 39, 3235 (2014).

[7] X. Zhang et al., Opt. Lett. 37, 1334 (2012).

[8] K. Krzempek, J. Sotor, K. Abramski, Opt. Lett. 41, 4995 (2016).

[9] G. Semaan, F. Ben Braham, J. Fourmont, M. Salhi, F. Bahloul, F. Sanchez, Opt. Lett. 41, 4767 (2016).

[10] K. Krzempek, K. Abramski, Opt. Expr. 24, 22379 (2016). 